TRANSACTIONS OF THE

AMERICAN MATHEMATICAL SOCIETY

Volume 364, Number 6, June 2012, Pages 3001-3021

S 0002-9947(2012)05391-8

Article electronically published on February 13, 2012

\title{
THE CANONICAL EXPANDING SOLITON AND HARNACK INEQUALITIES FOR RICCI FLOW
}

\author{
ESTHER CABEZAS-RIVAS AND PETER M. TOPPING
}

\begin{abstract}
We introduce the notion of Canonical Expanding Ricci Soliton, and use it to derive new Harnack inequalities for Ricci flow. This viewpoint also gives geometric insight into the existing Harnack inequalities of Hamilton and Brendle.
\end{abstract}

\section{INTRODUCTION}

Recently, in 4, we introduced the notion of Canonical Soliton. Roughly speaking, given any Ricci flow on a manifold $\mathcal{M}$ over a time interval $I \subset(-\infty, 0)$, we imagined the time direction as an additional space direction and constructed a shrinking Ricci soliton on $\mathcal{M} \times I$ with respect to a completely new time direction.

Considering these solitons in the context of known notions and theorems in Riemannian geometry then induced interesting concepts and results concerning the original Ricci flow, many of which were first discovered by Perelman [17. For example, considering geodesic distance in our soliton metrics gives rise to Perelman's $\mathcal{L}$-length.

It is also fruitful to consider existing Ricci flow theory applied to the Canonical Soliton flows. For example, applying the theory of McCann and the second author and Ilmanen [15] is one way of leading to the results of [19] which ultimately recovers essentially all of the monotonic quantities for Ricci flow used by Perelman [17. See [20] for a broader description.

In this paper we describe a slight variation of the Canonical Shrinking Solitons - namely the Canonical Expanding Solitons - which have completely different applications. These new solitons are adapted to explaining and proving Harnack inequalities in the spirit of the original result of Hamilton [10] and the more recent result of Brendle 2]. Our work recovers both of these known Harnack inequalities, and gives new ones too. (See Theorem 2.7.) In addition, our method explains clearly what is behind a Harnack inequality: it is simply the assertion that a given curvature condition is preserved on the Canonical Expanding Soliton.

As a by-product of our work we give an answer to the question of Wallach and Hamilton [11, which asks for a geometric construction whose curvature is represented by the matrix Harnack quantity of Hamilton [10. This question prompted the pioneering works of Chow-Chu [5] (see also the relevant modification in [7, chapter 11, §1.3]) and Chow-Knopf [6], which led in turn to the constructions of [17, §6] and [4. Each of these papers constructed an object whose curvatures were similar to the Hamilton's Harnack quantities, sometimes modulo either missing terms or valid for a different range of values of time as in Perelman's work [17, $\S 6, \S 7]$.

Received by the editors February 26, 2010 and, in revised form, June 4, 2010.

2010 Mathematics Subject Classification. Primary 53C44. 
It turns out that the Riemannian curvatures of the Canonical Expanding Solitons give rise to the exact Hamilton matrix Harnack quantities, with the closest parallels in the existing literature to be found within the approximation approach in [5, §4] and, more recently, the calculations of Brendle [2. In our case, for example, the full Hamilton-Harnack inequality is equivalent to the Canonical Expanding Soliton having weakly positive curvature operator (in an appropriate limit). Our new Harnack inequalities can be phrased in terms of this soliton satisfying other natural curvature conditions.

The paper is organised as follows. In Section 2 we introduce the Canonical Expanding Solitons and the flow they induce, describe their asymptotics and explain how they lead to Harnack inequalities. Our new Harnack inequalities are stated in Section 2.4. In Section 3 we give the rigorous proof of the Harnack inequalities stated in Theorem 2.7. To do that we use the Canonical Expanding Solitons to derive the equations satisfied by their (limiting) curvature $\mathcal{R}_{\infty}$, and then piece together an argument which borrows much from the work of Hamilton [10] and Brendle 2. The computations for the curvature of the Canonical Expanding Solitons are given in Appendix $\mathrm{A}$ and in Appendix $\mathrm{B}$ we describe a recent insight of Burkhard Wilking [21] which allows one to construct some new invariant curvature cones.

For an introduction to Ricci flow, we refer to [18; for further background on Canonical Solitons and an overview of the use of Harnack inequalities in Ricci flow, see 20 .

\section{The Canonical Expanding Solitons}

\subsection{Definitions and basic properties.}

Theorem 2.1. Suppose $g(t)$ is a Ricci flow, i.e. a solution of

$$
\frac{\partial g}{\partial t}=-2 \operatorname{Ric}(g(t))
$$

defined on a manifold $\mathcal{M}$ of dimension $n \in \mathbb{N}$, for $t$ within a time interval $[0, T]$, with uniformly bounded curvature. Suppose $N>0$, and define a metric $\check{g}_{N}$ (which we normally write simply as $\check{g})$ on $\check{\mathcal{M}}:=\mathcal{M} \times(0, T]$ by

$$
\check{g}_{i j}=\frac{g_{i j}}{t}, \quad \check{g}_{00}=\frac{N}{2 t^{3}}+\frac{R}{t}+\frac{n}{2 t^{2}}, \quad \check{g}_{0 i}=0,
$$

where $i, j$ are coordinate indices on the $\mathcal{M}$ factor, 0 represents the index of the time coordinate $t \in(0, T]$, and the scalar curvature of $g$ is written as $R$.

Then up to errors of order $\frac{1}{N}$, the metric $\check{g}$ is a gradient expanding Ricci soliton on the higher dimensional space $\check{\mathcal{M}}$ :

$$
E_{N}:=\operatorname{Ric}(\check{g})+\operatorname{Hess}_{\check{g}}\left(-\frac{N}{2 t}\right)+\frac{1}{2} \check{g} \simeq 0,
$$

by which we mean that for any $k \in\{0,1,2, \ldots\}$ the quantity

$$
N\left[\check{\nabla}^{k} E_{N}\right]
$$

is bounded uniformly locally on $\check{\mathcal{M}}$ (independently of $N$ ), where $\check{\nabla}$ is the Levi-Civita connection corresponding to $\check{g}$. 
Remark 2.2. Implicit in this theorem is that $\check{g}_{N}$ is a Riemannian metric, i.e. positive definite, for any $N>0$. This is because the maximum principle applied to $R$ implies that $R \geq-\frac{n}{2 t}$. The same argument on closed manifolds can be found in [18. Corollary 3.2.5]; the maximum principle is valid for our possibly noncompact Ricci flows since we are assuming uniformly bounded curvature (see, for example, [7. Corollary 7.43]). One further consequence of the fact that $R+\frac{n}{2 t} \geq 0$ is that the mean curvature of any submanifold $\mathcal{M} \times\{t\}$ of $(\check{\mathcal{M}}, \check{g})$ has a sign (cf. 4, Section 6]) although we do not pursue that here.

This construction should be compared to the Canonical Shrinking Solitons of 4 . Various signs have changed, and every $\tau$ has been replaced with a $t$. We have also strengthened the sense in which (2.2) is to hold in order to make it more useful in rigorous proofs, and considered Ricci flows defined all the way down to $t=0$ as is relevant in the study of Harnack estimates.

The proof reduces to computing all relevant quantities explicitly. We give the results of these computations, including exact expressions for the full curvature tensor of $\check{g}$ in Appendix A,

We now introduce a new time parameter $s \in(0,1]$ and consider the flow of metrics

$$
G(s):=s \psi_{s}^{*}(\check{g})
$$

where $\psi_{s}: \check{\mathcal{M}} \rightarrow \check{\mathcal{M}}$ is the family of maps, diffeomorphic onto their images, generated by integrating the collection of vector fields

$$
\begin{aligned}
X_{s} & :=\frac{1}{s} \check{\nabla}\left(-\frac{N}{2 t}\right) \\
& =\frac{t}{s} \frac{\partial}{\partial t}-\check{g}^{00}\left(R+\frac{n}{2 t}\right) \frac{1}{s} \frac{\partial}{\partial t}
\end{aligned}
$$

starting with $\psi_{1}=i d e n t i t y$. If we imagine $N$ to be large, then $X_{s}$ is approximately the vector field $\frac{t}{s} \frac{\partial}{\partial t}$, which could be integrated on the whole of $\mathcal{M} \times(0, \infty)$ to give the map $\psi_{s}^{\infty}: \mathcal{M} \times(0, \infty) \rightarrow \mathcal{M} \times(0, \infty)$ defined by

$$
\psi_{s}^{\infty}(x, t)=(x, s t) .
$$

(For arbitrary $N>0$, we can compare $X_{s}$ and $\frac{t}{s} \frac{\partial}{\partial t}$ using (2.4) because $R+\frac{n}{2 t} \geq 0$ as in Remark 2.2. and thus one can check that the maps $\psi_{s}$ are well-defined.) A slightly closer inspection yields:

Proposition 2.3. The map $\psi_{s}$ converges smoothly in its arguments $x \in \mathcal{M}, t \in$ $(0, T]$ and $s \in(0,1]$ to (an appropriate restriction of) $\psi_{s}^{\infty}$ as $N \rightarrow \infty$.

If $\check{g}$ were an exact Ricci soliton metric, then $G(s)$ would be an exact Ricci flow on $\mathcal{M} \times(0, T]$ for $s \in(0,1]$ (see [18, $\S 1.2 .2]$ ). A minor adjustment of the standard theory reveals:

Proposition 2.4. The flow $G(s)$ satisfies

$$
\frac{\partial G}{\partial s}=-2 \operatorname{Ric}(G(s))+\psi_{s}^{*}\left(E_{N}\right)
$$

where $E_{N}$ is defined in Theorem 2.1.

Since $G(s)$ is essentially a Ricci flow, we can imagine that curvature conditions such as positive curvature operator which are preserved for Ricci flows might be essentially preserved for $G(s)$. If we can argue that $G(s)$ satisfies such a condition 
in the limit $s \downarrow 0$, then we should deduce the same condition for $G(1)=\check{g}$, and the idea is that a curvature condition for $\check{g}$ is the geometric way of expressing a Harnack inequality. We will see precise assertions along these lines later, but for now, this reasoning justifies the following section.

2.2. Asymptotics of the canonical expanding soliton. We will argue that in the limit $t \downarrow 0$, the Canonical Solitons are conical. Near $t=0$, the dominant term in the definition of $\check{g}_{00}$ is emphatically $\frac{N}{2 t^{3}}$. If we neglect the other terms for the moment, and change variables from $t$ to $r:=t^{-\frac{1}{2}}$, then for large $r$ (small $t$ ) $\check{g}$ can be written approximately as

$$
\begin{aligned}
\check{g}_{N} & \sim \frac{g(t)}{t}+\frac{N}{2 t^{3}} d t^{2} \\
& =r^{2} g\left(r^{-2}\right)+2 N d r^{2}
\end{aligned}
$$

and this suggests that asymptotically the Canonical Soliton opens like the cone

$$
\Sigma_{N}:=\left(\mathcal{M} \times(0, \infty), \frac{g(0)}{t}+\frac{N}{2 t^{3}} d t^{2}\right)=\left(\mathcal{M} \times(0, \infty), r^{2} g(0)+2 N d r^{2}\right)
$$

(using coordinates $(x, t)$ or $(x, r)$ respectively on $\mathcal{M} \times(0, \infty))$ with shallow cone angle for large $N$. This motivates:

Lemma 2.5. If we define $\bar{G}(s):=s\left(\psi_{s}^{\infty}\right) *\left(\check{g}_{N}\right)$ on $\mathcal{M} \times(0, T / s]$, then

$$
\bar{G}(s) \rightarrow \frac{g(0)}{t}+\frac{N}{2 t^{3}} d t^{2}
$$

smoothly locally on $\mathcal{M} \times(0, \infty)$ as $s \downarrow 0$.

Proof. It may be clearest to make a third change of coordinates, writing $\alpha=\ln t$. Then

$$
\frac{g(0)}{t}+\frac{N}{2 t^{3}} d t^{2}=e^{-\alpha}\left(g(0)+\frac{N}{2} d \alpha^{2}\right)
$$

and the map $\psi_{s}^{\infty}$, viewed in $(x, \alpha)$ coordinates as a map $\mathcal{M} \times \mathbb{R} \rightarrow \mathcal{M} \times \mathbb{R}$, corresponds to

$$
\psi_{s}^{\infty}(x, \alpha)=(x, \alpha+\ln s)
$$

Moreover,

$$
\check{g}_{N}=e^{-\alpha}\left(g\left(e^{\alpha}\right)+\frac{N}{2} d \alpha^{2}\right)+\left(R e^{\alpha}+\frac{n}{2}\right) d \alpha^{2}
$$

and so

$$
\bar{G}(s)=e^{-\alpha}\left(g\left(e^{\alpha+\ln s}\right)+\frac{N}{2} d \alpha^{2}\right)+\left(R s^{2} e^{\alpha}+s \frac{n}{2}\right) d \alpha^{2} .
$$

One consequence of this is that for any $x \in \mathcal{M}$ and sequence $t_{i} \downarrow 0$, we have convergence of pointed rescalings of the Canonical Expanding Soliton:

$$
\left(\check{\mathcal{M}}, t_{i} \check{g}_{N},\left(x, t_{i}\right)\right) \rightarrow\left(\Sigma_{N},(x, \alpha=0)\right)
$$

as $i \rightarrow \infty$ in the sense of Cheeger-Gromov convergence [18, $\S 7$ ]. It is also relevant to note that

as $N \rightarrow \infty$, where

$$
\left(\Sigma_{N},(x, \alpha=0)\right) \rightarrow\left(\Sigma_{\infty},(x, 0)\right)
$$

$$
\Sigma_{\infty}:=(\mathcal{M}, g(0)) \times \mathbb{R}
$$


i.e., the cone straightens out to a cylinder. In practice, we will be most interested in taking limits of geometric quantities as $N \rightarrow \infty$ (despite the fact that the metric itself will degenerate) and then as $s \downarrow 0$ rather than the other way around.

2.3. Using canonical expanding solitons to give Harnack inequalities. We are now in a position to elaborate on the use of Theorem 2.1 to obtain Harnack estimates. It is well known that certain curvature conditions are preserved under Ricci flow. For example, a Ricci flow on a closed manifold which starts with weakly positive curvature operator will also satisfy this property at later times.

Given a Ricci flow for which we would like a Harnack inequality, the trick, effectively, is to apply this preservation principle not to the Ricci flow itself, but to its Canonical Expanding Soliton. Given the asymptotics discussed in the previous section, we should study Ricci flows $g(t)$ on an $n$-manifold $\mathcal{M}$, for which the curvature of $(\mathcal{M}, g(0)) \times \mathbb{R}$ lies in a subspace of the space of all possible curvatures which is preserved under $(n+1)$-dimensional Ricci flow. If we were to make the leap of faith that such a curvature condition should also be preserved under the approximate Ricci flow generated by the (approximate, incomplete) Canonical Soliton, then we would deduce that $G(1)=\check{g}$ should satisfy this condition, and this statement can be considered to be a Harnack inequality.

In order to make a precise statement of the Harnack inequalities, we will pass to the limit $N \rightarrow \infty$. The Canonical Soliton metrics $\check{g}_{N}$ degenerate in this limit, although if we view them as metrics on $T^{*} \check{\mathcal{M}}$ rather than on $T \check{\mathcal{M}}$, then they converge to a weakly positive definite tensor $\check{g}_{\infty} \in S y m^{2}(T \check{\mathcal{M}})$ whose only nonzero components are $\left(\check{g}_{\infty}\right)^{i j}=t g^{i j}$. More importantly, some of the geometric quantities such as curvature associated with $\check{g}_{N}$ behave well in the same limit, and the actual Harnack estimates will be statements about them.

Let $V$ be a (real) vector space of dimension $m$. We call $\mathcal{R} \in \otimes^{4} V^{*}$ an algebraic curvature tensor if it satisfies the symmetries of the curvature tensor of a Riemannian metric, including the first Bianchi identity (cf. Chapter V of [13]). Given a manifold $\mathcal{N}^{m}$, we use the same terminology to describe a section of $\otimes^{4} T^{*} \mathcal{N}$ which is an algebraic curvature tensor in each fibre.

Proposition 2.6. In the setting of Theorem 2.1, the full curvature tensor $\mathcal{R}\left(\check{g}_{N}\right)$ of $\check{g}_{N}$, viewed as a section of $\otimes{ }^{4} T^{*} \check{\mathcal{M}}$, converges smoothly as $N \rightarrow \infty$ to a limit algebraic curvature tensor $\mathcal{R}_{\infty}$. At a point $(x, t)$ in $\check{\mathcal{M}}$, the coefficients of the tensor $\mathcal{R}_{\infty}$ are given in terms of the coefficients of the curvature of $g(t)$ at the point $x$ by

$$
\begin{aligned}
& \stackrel{\infty}{R}_{i j k l}=\frac{1}{t} R_{i j k l}, \\
& \stackrel{\infty}{R}_{i 0 j 0}=\frac{1}{t}\left(\Delta R_{i j}+2 R_{i k j l} R^{k l}-R_{i}^{k} R_{j k}+\frac{R_{i j}}{2 t}-\frac{1}{2} \nabla_{i} \nabla_{j} R\right), \\
& \stackrel{\infty}{R}_{i j 0 k}=\frac{1}{t}\left(\nabla_{i} R_{j k}-\nabla_{j} R_{i k}\right) .
\end{aligned}
$$

Moreover, the Ricci curvature $\operatorname{Ric}\left(\check{g}_{N}\right) \in \Gamma\left(\operatorname{Sym}^{2} T^{*} \check{\mathcal{M}}\right)$ converges to a limit $\operatorname{Ric}_{\infty}$ determined by

$$
\operatorname{Ric}_{\infty}\left(X+\frac{\partial}{\partial t}, X+\frac{\partial}{\partial t}\right)=\operatorname{Ric}_{g(t)}(X, X)+\langle X, \nabla R\rangle_{g(t)}+\frac{1}{2}\left(\frac{\partial R}{\partial t}+\frac{R}{t}\right),
$$

for each $X \in T \mathcal{M}$. 
The proposition follows immediately from Appendix $\mathrm{A}$.

An algebraic curvature tensor with the same coefficients as $\mathcal{R}_{\infty}$ arises as the limit (as $\varepsilon \rightarrow \infty$ and $\delta \rightarrow 0$ ) of the Riemannian curvature associated with the two-parameter family of Riemannian metrics $\tilde{g}_{\varepsilon, \delta}$ introduced in [5, §4]. Compare also with the definition of the $(0,4)$-tensor $S$ in 2 .

The curvature also converges when viewed as any tensor of type $(p, q)$ with $p+q=4$, but because the metric $\check{g}_{N}$ degenerates in the limit, the assertion above carries the most information.

The symmetries of an algebraic curvature tensor on a vector space $V$, say, allow one to see it as a symmetric bilinear form on $\Lambda^{2} V$, constrained further by the Bianchi identity. We sometimes emphasise this viewpoint by calling such a constrained element of $\operatorname{Sym}^{2}\left(\Lambda^{2} V^{*}\right)$ an (algebraic) curvature (bilinear) form, and write the entire space of such forms as $\operatorname{Sym}_{B}^{2}\left(\Lambda^{2} V^{*}\right)$. We will occasionally use the same notation for the space of algebraic curvature tensors, that is, we will switch between these two viewpoints implicitly, often without changing notation.

Viewing $\mathcal{R}_{\infty}$ as a section of $\operatorname{Sym}_{B}^{2}\left(\Lambda^{2} T^{*} \check{\mathcal{M}}\right.$ ) (using the obvious extension of the notation above) the full matrix Harnack inequality of Hamilton [10] is precisely equivalent to $\mathcal{R}_{\infty}$ being (weakly) positive definite. (This would normally be referred to as weakly positive curvature operator, but as mentioned above, because the limit metric $\check{g}_{\infty}$ is degenerate, that would be a weaker assertion.) Hamilton's trace Harnack inequality is precisely equivalent to $\mathrm{Ric}_{\infty}$ being weakly positive definite. Brendle's Harnack inequality 2 is precisely equivalent to $\mathcal{R}_{\infty}$ lying in a certain cone introduced by Brendle and Schoen [1] (see Appendix B).

Finally, if one were to assume that all Harnack inequalities arose in this way, one would conclude that the long-sought Harnack inequality for Ricci flows $g(t)$ on 3manifolds with weakly positive Ricci curvature would be unreasonable. One would deduce that $(\mathcal{M}, g(0)) \times \mathbb{R}$ has weakly positive Ricci curvature, but the framework of this paper would then require weakly positive Ricci curvature to be preserved for 4-dimensional Ricci flows, which is false (cf. 14 and 12 for the compact and noncompact case, respectively).

In the next section we find some other preserved curvature conditions which yield new Harnack inequalities, and in Section 2.5 we explain to what extent one might expect to generalise further.

2.4. New Harnack inequalities. As we have indicated, our Harnack inequalities will be phrased in terms of the limiting curvature form $\mathcal{R}_{\infty}$ lying in appropriate subsets of $\operatorname{Sym}_{B}^{2}\left(\Lambda^{2} T^{*} \mathcal{\mathcal { M }}\right)$. We now work towards defining some examples of such subsets.

Following [16] and 21] we consider the complexified tangent bundle $T^{\mathbb{C}} \mathcal{N}$ of a manifold $\mathcal{N}$, and implicitly extend each curvature form complex linearly to act on complexified 2-vectors - i.e. elements of $\Lambda^{2} T^{\mathbb{C}} \mathcal{N}$. To such a 2-vector $\omega$, one can associate a rank defined to be the least number $k \in\{0,1,2, \ldots\}$ such that in each fibre we can write $\omega$ as a complex linear combination of at most $k$ simple elements $u \wedge v$ with $u, v \in T^{\mathbb{C}} \mathcal{N}$.

With this viewpoint, we can define the following convex cones within $\operatorname{Sym}_{B}^{2}\left(\Lambda^{2} T^{*} \mathcal{N}\right)$, which arise from 'Wilking' cones as discussed in Appendix B.

$$
\begin{aligned}
\mathcal{C}_{k}(\mathcal{N}):=\left\{\mathcal{R} \in \operatorname{Sym}_{B}^{2}\left(\Lambda^{2} T^{*} \mathcal{N}\right) \mid \mathcal{R}(\omega, \bar{\omega}) \geq 0\right. \\
\left.\quad \text { for all } \omega \in \Lambda^{2} T^{\mathbb{C}} \mathcal{N} \text { of rank no more than } k\right\} .
\end{aligned}
$$


We will see that these cones are invariant under Ricci flow in the sense that if $g(t)$ is a Ricci flow on a closed manifold $\mathcal{M}$ for $t \in[0, T]$ and $\mathcal{R}(g(0)) \in \mathcal{C}_{k}(\mathcal{M})$, then $\mathcal{R}(g(t)) \in \mathcal{C}_{k}(\mathcal{M})$ for all $t \in[0, T]$. Note that the following theorem does not require $\mathcal{M}$ to be closed.

Theorem 2.7 (Main Harnack Theorem). Suppose $g(t)$ is a complete Ricci flow on a manifold $\mathcal{M}$ for $t \in(0, T]$ with scalar curvature uniformly bounded from above, and for some $k \in \mathbb{N}, \mathcal{R}(g(t)) \in \mathcal{C}_{k}(\mathcal{M})$ for all $t \in(0, T]$. Then $\mathcal{R}_{\infty} \in \mathcal{C}_{k}(\check{\mathcal{M}})$.

Remark 2.8. Note that the cones $\mathcal{C}_{k}$ are nested in the sense that if $k_{1} \leq k_{2}$, then $\mathcal{C}_{k_{1}}(\mathcal{N}) \supset \mathcal{C}_{k_{2}}(\mathcal{N})$. Moreover, if $\mathcal{R}_{\infty} \in \mathcal{C}_{k}(\check{\mathcal{M}})$ for some $k$ (even $k=1$ ), then $\operatorname{Ric}_{\infty}$ is positive definite, so we deduce Hamilton's trace Harnack inequality. By definition, a metric whose curvature tensor lies in $\mathcal{C}_{1}(\mathcal{N})$ has weakly positive sectional curvature, so the hypothesised upper bound for the scalar curvature implies a uniform upper and lower bound on the full curvature tensor.

The highest possible rank of an element $\omega \in \Lambda^{2} T \mathcal{N}$ is $\left[\frac{\operatorname{dim}(\mathcal{N})}{2}\right]$, and therefore if $k=\left[\frac{n+1}{2}\right]$, the cone $\mathcal{C}_{k}(\check{\mathcal{M}})$ is precisely the weakly positive definite elements of $\operatorname{Sym}_{B}^{2}\left(\Lambda^{2} T^{*} \check{\mathcal{M}}\right)$, and we recover the main result of [10] stated in a geometric form:

Corollary 2.9 (Equivalent to Hamilton [10, Main Theorem]). Suppose $g(t)$ is a complete Ricci flow on a manifold $\mathcal{M}$ for $t \in(0, T]$ with uniformly bounded curvature. If $\mathcal{R}(g(t))$ is weakly positive definite for all $t \in(0, T]$, then $\mathcal{R}_{\infty}$ is weakly positive definite.

The fact that the cone of weakly positive definite curvature forms is preserved under Ricci flow is also, separately, due to Hamilton [9].

The special case $k=1$ is the main result of [2]:

Corollary 2.10 (Equivalent to Brendle [2, Proposition 9]). Suppose $g(t)$ is a complete Ricci flow on a manifold $\mathcal{M}$ for $t \in(0, T]$ with scalar curvature uniformly bounded from above. If $\mathcal{R}(g(t)) \in \mathcal{C}_{1}(\mathcal{M})$ for all $t \in(0, T]$, then $\mathcal{R}_{\infty} \in \mathcal{C}_{1}(\mathcal{M})$.

Note that the main result 2, Theorem 1] as stated is a little weaker than this corollary, but it is deduced from [2, Proposition 9], which is just as strong. The preserved cone $\mathcal{C}_{1}$ was discovered by Brendle and Schoen [1] and was originally described as the cone of curvature forms of manifolds $(\mathcal{N}, g)$ for which $(\mathcal{N}, g) \times \mathbb{R}^{2}$ has weakly positive isotropic curvature. The $\mathcal{C}_{1}$ formulation has the advantage of emphasising that the cone is independent of the background metric (a fact appreciated by Brendle in [2]).

For intermediate $k$, Theorem 2.7 can be considered to be some sort of interpolation between these two corollaries. One has weaker hypotheses than Hamilton's result, and stronger conclusions than Brendle's result (but weaker conclusions than Hamilton's result, and stronger hypotheses than Brendle's result).

In the bigger scheme of things, Hamilton's result has been crucial in the study of 3-manifolds using Ricci flow. There, the blow-ups of singularities do satisfy the generally rather restrictive hypothesis of weakly positive curvature form, although the most useful conclusion is the trace Harnack result (Remark 2.8). Brendle's result, having the weakest hypothesis, currently holds the most promise for applications to the study of manifolds of positive isotropic curvature [16] via Ricci flow.

Remark 2.11. Although we have stated Theorem 2.7 for the cones $\mathcal{C}_{k}$, the same proof works for more general Wilking cones (see Appendix B) with the additional 
assumption that they can be defined independently of a background metric. Moreover, the heuristics suggest that the result holds for more general convex cones which are invariant under the Ricci flow, as we describe in the next section, although we do not currently know of any suitable cones $K$ other than those already dealt with directly in this paper.

2.5. More general cones. We want to imagine more general convex cones $K$ within $\operatorname{Sym}_{B}^{2}\left(\Lambda^{2} V^{*}\right)$, with $\operatorname{dim} V=n+1$, so that the assertion of a Harnack inequality will be that $\mathcal{R}_{\infty} \in K$. (Recall that a convex cone $K$ in a vector space is a subset such that if $\lambda \geq 0$ and $a, b \in K$, then $\lambda a \in K$ and $a+b \in K$.) Given the explanations of previous sections, we would like $K$ to be an invariant cone under Ricci flow, and since the metric $\check{g}_{\infty}$ is degenerate, it will be appropriate to ask that the cone is $G L(V)$-invariant. To clarify both notions:

Definition 2.12. A cone $K$ within the vector space $S y m_{B}^{2}\left(\Lambda^{2} V^{*}\right)$ of algebraic curvature tensors on an $m$-dimensional real vector space $V$ is called $G L(V)$-invariant if for each $T \in K$ and linear map $A \in G L(V)$, the tensor $T_{A} \in S y m_{B}^{2}\left(\Lambda^{2} V^{*}\right)$ defined by

$$
T_{A}\left(v_{1}, v_{2}, v_{3}, v_{4}\right):=T\left(A v_{1}, A v_{2}, A v_{3}, A v_{4}\right)
$$

also lies in $K$.

Such a $G L(V)$-invariant cone $K$ that is also closed and convex, is said to be invariant under Ricci flow if when we define, for $T \in S y m_{B}^{2}\left(\Lambda^{2} V^{*}\right)$ and positive definite $g \in S y m^{2} V$, the tensor

$$
Q(T, g)_{a b c d}:=2 g^{\alpha \gamma} g^{\beta \delta}\left[T_{a \alpha b \beta} T_{c \gamma d \delta}-T_{a \alpha b \beta} T_{d \gamma c \delta}+T_{a \alpha c \beta} T_{b \gamma d \delta}-T_{a \alpha d \beta} T_{b \gamma c \delta}\right]
$$

then for all $T \in \partial K$ and all (equivalently one) $g$, the tensor $Q(T, g)$ points into the interior of $K$ at $T$. That is, for any $\Upsilon \in\left(\operatorname{Sym}_{B}^{2}\left(\Lambda^{2} V^{*}\right)\right)^{*}$ such that $\Upsilon(\tilde{T}-T)>0$ implies $\tilde{T} \notin K$, we have $\Upsilon(Q(T, g)) \leq 0$. Or, alternatively phrased (see [9, Lemma 4.1] for a proof of the equivalence), the cone $K$ is preserved under the ODE $\dot{T}=$ $Q(T, g)$ (for any or all $g$ ).

Note that we can talk about a curvature form/tensor $\mathcal{R}$ on a manifold $\mathcal{N}^{m}$ lying in a $G L(V)$-invariant cone $K$ : we ask that at each point $x \in \mathcal{N}$, after identifying $T_{x} \mathcal{N}$ with $V$ via an arbitrary linear bijection, we have $\mathcal{R}(x) \in K$.

The terminology of the definition is justified because Hamilton's ODE-PDE theorem (see Theorem 4.3 in [9]) tells us that if $g(t)$ is a Ricci flow on a closed manifold such that $\mathcal{R}(g(0))$ lies in a cone $K$ as in Definition 2.12 , then $\mathcal{R}(g(t)) \in K$ for all later times $t$.

Conjecture 2.13. Let $V$ be a vector space of dimension $n+1$. Suppose $K$ is a closed $G L(V)$-invariant convex cone within $\operatorname{Sym}_{B}^{2}\left(\Lambda^{2} V^{*}\right)$ which contains all weakly positive definite curvature forms, and is invariant under the Ricci flow in the sense of Definition 2.12. Suppose further that $g(t)$ is a Ricci flow on a manifold $\mathcal{M}^{n}$ for $t \in(0, T]$ with uniformly bounded curvature, and $\mathcal{R}((\mathcal{M}, g(t)) \times \mathbb{R}) \in K$ for all $(x, t) \in \check{\mathcal{M}}$. Then $\mathcal{R}_{\infty} \in K$.

It would be particularly interesting in this context to find such a $G L\left(V^{n+1}\right)$ invariant cone containing the cone $\mathcal{C}\left(S_{1}\right)$ of Appendix B. but contained in the cone of curvature operators with weakly positive sectional curvature. 


\section{Proof of the Harnack estimates}

In this section we give a rigorous proof of Theorem 2.7 following the ideas of the previous section, and the earlier papers of Hamilton [10] and Brendle [2].

3.1. Applying the canonical expanding soliton to derive equations satisfied by $\mathcal{R}_{\infty}$. We have seen how the curvature of the metrics $\check{g}_{N}$ converges to $\mathcal{R}_{\infty}$ as $N \rightarrow \infty$, and in this section we will apply what we know about the curvatures of Ricci flows to derive information about this limit. More precisely, we want to derive a parabolic-type equation (3.7) which it satisfies. We will also have to consider one further object which behaves well in the same limit, namely the connection.

Proposition 3.1. In the setting of Theorem 2.1, the Levi-Civita connection $\check{\nabla}$ of $\check{g}_{N}$ converges smoothly as $N \rightarrow \infty$ to a limit $\stackrel{\infty}{\nabla}$. More precisely, after choosing local coordinates $\left\{x_{1}, \ldots, x^{n}, t\right\}$ near some point in $\check{\mathcal{M}}$, the Christoffel symbols with respect to those coordinates will converge smoothly. At a point $(x, t)$ in $\check{\mathcal{M}}$, the Christoffel symbols of $\stackrel{\infty}{\nabla}$ are given in terms of the coefficients of $g(t)$ and its curvature at the point $x$ by

$$
\begin{aligned}
& \stackrel{\infty}{\Gamma}_{j k}^{i}=\Gamma_{j k}^{i} ; \quad \stackrel{\infty}{\Gamma_{j 0}^{i}}=-\left(R^{i}{ }_{j}+\frac{\delta^{i}{ }_{j}}{2 t}\right) ; \quad \stackrel{\infty}{\Gamma_{00}^{i}}=-\frac{1}{2} g^{i j} \frac{\partial R}{\partial x^{j}} ; \\
& \stackrel{\infty}{\Gamma}_{j k}^{0}=0 ; \quad \stackrel{\infty}{\Gamma}{ }_{i 0}^{0}=0 ; \quad \stackrel{\infty}{\Gamma}_{00}^{0}=-\frac{3}{2 t} .
\end{aligned}
$$

The proof is immediate from Appendix $\mathrm{A}$.

The above connection $\stackrel{\infty}{\nabla}$ also arises in Brendle [2]. Connections with similar Christoffel symbols can be found in [5] (see also the modified version in [7]) and [6].

This connection also induces a limiting Laplacian

$$
\Delta_{t}:=g^{i j} \stackrel{\infty}{\nabla} i \stackrel{\infty}{\nabla},
$$

which can be applied to any tensor on $\check{\mathcal{M}}$. Because $\stackrel{\infty}{\Gamma}_{i j}^{0}=0$, we readily check the following assertion.

Lemma 3.2. If $f: \check{\mathcal{M}} \rightarrow \mathbb{R}$, then

$$
\Delta_{t} f=\Delta_{g(t)}[f(\cdot, t)] .
$$

In particular, for any tensor $S$ on $\check{\mathcal{M}}$ and $F: \mathbb{R} \rightarrow \mathbb{R}$, we have $\Delta_{t}(F(t) S)=$ $F(t) \Delta_{t} S$.

Now recall (see for example [18, Proposition 2.5.1]) that the curvature $\mathcal{R}$ of a Ricci flow $g(t)$ satisfies the equation

$$
\frac{\partial \mathcal{R}}{\partial t}=\Delta \mathcal{R}+F(\mathcal{R}, g)+Q(\mathcal{R}, g)
$$

where $Q$ was defined as in (2.6) and $F$ is a map of the same type defined by

$$
\begin{aligned}
F(T, g)_{a b c d}: & =-g^{\alpha \beta} g^{\gamma \delta}\left[T_{\alpha b c d} T_{a \gamma \beta \delta}+T_{a \alpha c d} T_{b \gamma \beta \delta}+T_{a b \alpha d} T_{c \gamma \beta \delta}+T_{a b c \alpha} T_{d \gamma \beta \delta}\right] \\
& =-g^{\alpha \beta}\left[T_{\alpha b c d} T_{a \beta}+T_{a \alpha c d} T_{b \beta}+T_{a b \alpha d} T_{c \beta}+T_{a b c \alpha} T_{d \beta}\right],
\end{aligned}
$$

where $T_{a b}=g^{c d} T_{a c b d}$. 
As usual, we wish to apply this type of flow equation not to the Ricci flow $g(t)$ under consideration, but to the flow $G(s)$ of its Canonical Soliton $\check{g}_{N}$. By Theorem 2.1. Proposition 2.3 and Proposition 2.4 we have

$$
\frac{\partial G}{\partial s} \simeq-2 \operatorname{Ric}(G(s))
$$

where $\simeq$ is used in the precise sense of Theorem 2.1 that is, the difference of the left-hand side and the right-hand side can be differentiated at will using $\check{\nabla}$, and multiplied by $N$, and will still remain locally bounded as $N \rightarrow \infty$. Therefore, a glance at the derivation of (3.2) shows that in fact

$$
\frac{\partial \mathcal{R}_{G(s)}}{\partial s} \simeq \Delta_{G(s)} \mathcal{R}_{G(s)}+F\left(\mathcal{R}_{G(s)}, G(s)\right)+Q\left(\mathcal{R}_{G(s)}, G(s)\right) .
$$

We may now set $s=1$ and take the limit $N \rightarrow \infty$. Clearly

$$
F\left(\mathcal{R}_{G(1)}, G(1)\right)=F\left(\mathcal{R}_{\check{g}_{N}}, \check{g}_{N}\right) \rightarrow F\left(\mathcal{R}_{\infty}, \check{g}_{\infty}\right)
$$

and

as $N \rightarrow \infty$. Meanwhile,

$$
Q\left(\mathcal{R}_{G(1)}, G(1)\right)=Q\left(\mathcal{R}_{\check{g}_{N}}, \check{g}_{N}\right) \rightarrow Q\left(\mathcal{R}_{\infty}, \check{g}_{\infty}\right)
$$

$$
\begin{aligned}
\frac{\partial \mathcal{R}_{G(s)}}{\partial s} & =\frac{\partial}{\partial s}\left[s \mathcal{R}_{\psi_{s}^{*}(\breve{g})}\right]=\frac{\partial}{\partial s}\left[s \psi_{s}^{*}\left(\mathcal{R}_{\breve{g}}\right)\right] \\
& =\psi_{s}^{*}\left[\mathcal{R}_{\breve{g}}+s \mathcal{L}_{\frac{1}{s} \check{\nabla}\left(-\frac{N}{2 t}\right)} \mathcal{R}_{\breve{g}}\right] \\
& \simeq \psi_{s}^{*}\left[\mathcal{R}_{\breve{g}}+\mathcal{L}_{t \frac{\partial}{\partial t}} \mathcal{R}_{\breve{g}}\right]
\end{aligned}
$$

by (2.4). Evaluating at $s=1$ (where $\psi_{1}$ is the identity) we find that

$$
\left.\frac{\partial \mathcal{R}_{G(s)}}{\partial s}\right|_{s=1} \rightarrow \mathcal{R}_{\infty}+\mathcal{L}_{t \frac{\partial}{\partial t}} \mathcal{R}_{\infty}
$$

as $N \rightarrow \infty$. Finally,

$$
\Delta_{G(1)} \mathcal{R}_{G(1)}=\Delta_{\check{g}} \mathcal{R}_{\check{g}}=\check{g}^{i j} \check{\nabla}_{i} \check{\nabla}_{j} \mathcal{R}_{\check{g}}+\check{g}^{00} \check{\nabla}_{0} \check{\nabla}_{0} \mathcal{R}_{\check{g}}
$$

and therefore

$$
\Delta_{G(1)} \mathcal{R}_{G(1)} \rightarrow t \Delta_{t} \mathcal{R}_{\infty},
$$

where $\Delta_{t}$ is from (3.1).

Combining all these formulae and passing to the limit in (3.4), we find the desired formula

$$
\mathcal{L}_{t \frac{\partial}{\partial t}} \mathcal{R}_{\infty}=t \Delta_{t} \mathcal{R}_{\infty}+F\left(\mathcal{R}_{\infty}, \check{g}_{\infty}\right)+Q\left(\mathcal{R}_{\infty}, \check{g}_{\infty}\right)-\mathcal{R}_{\infty} .
$$

3.2. Being on the boundary of a $G L(V)$-invariant cone. Suppose we have a convex cone $K$ in the space of algebraic curvature forms which is $G L(V)$-invariant in the sense of Definition 2.12 In this section we show some useful identities which hold whenever we are on the boundary of $K$, which will imply that the second term on the right-hand side of (3.7) points into the interior of $K$ when $\mathcal{R}_{\infty} \in \partial K$. If we assume that $K$ is invariant under Ricci flow (again in the sense of Definition 2.12), then the third term on the right-hand side of (3.7) points into the interior of $K$ when $\mathcal{R}_{\infty} \in \partial K$. Since $K$ is a cone, the same is true for the fourth term on the right-hand side of (3.7). One can speculate then that an appropriate ODE-PDE 
theorem (similar to that proved by Hamilton in [9]) applied to $\mathcal{R}_{\infty}$, viewed as an appropriate time-dependent section of a bundle over $\mathcal{M}$, would tell us that the PDE (3.7) would leave $K$ invariant, and that would give a proof of Conjecture 2.13.

To avoid having to prove an appropriate ODE-PDE theorem (handling noncompact manifolds and without a fixed underlying bundle metric) we will take a more direct route in this paper.

Lemma 3.3. If $\Theta$ is an algebraic curvature tensor on the boundary of a $G L(V)$ invariant cone $K \subset \operatorname{Sym}_{B}^{2}\left(\Lambda^{2} V^{*}\right)$, then for any $v_{1}, \ldots, v_{4} \in V$ and endomorphism $A \in V \otimes V^{*}$, the tensor

$$
\begin{aligned}
\left(v_{1}, v_{2}, v_{3}, v_{4}\right) \mapsto & \Theta\left(A v_{1}, v_{2}, v_{3}, v_{4}\right)+\Theta\left(v_{1}, A v_{2}, v_{3}, v_{4}\right) \\
& +\Theta\left(v_{1}, v_{2}, A v_{3}, v_{4}\right)+\Theta\left(v_{1}, v_{2}, v_{3}, A v_{4}\right)
\end{aligned}
$$

points into the interior of $K$. Moreover, it points along the boundary in the sense that its negation also points into the interior.

Proof. If $\Theta \in \partial K$, then $\Theta_{\alpha}$ defined for $\alpha \in \mathbb{R}$ near 0 by

$$
\Theta_{\alpha}\left(v_{1}, v_{2}, v_{3}, v_{4}\right):=\Theta\left((I+\alpha A) v_{1},(I+\alpha A) v_{2},(I+\alpha A) v_{3},(I+\alpha A) v_{4}\right)
$$

must also lie in $\partial K$ by virtue of the $G L(V)$-invariance. Differentiating with respect to $\alpha$ at $\alpha=0$ gives the first assertion, and the rest follows by changing the sign of $A$.

As a particular case, we have that $F\left(\Theta, \check{g}_{\infty}\right)$ as defined in (3.3) points along the boundary of $K$. Accordingly,

Corollary 3.4. If the cone $K$ from Lemma 3.3 is also a Wilking Cone $\mathcal{C}(S)$ (see Appendix (B) and $\operatorname{dim} V=n+1$, then

$$
F\left(\Theta, \check{g}_{\infty}\right)(\omega, \bar{\omega})=0 \quad \text { whenever } \Theta(\omega, \bar{\omega})=0 \text { for } \omega \in \Lambda^{2}\left(V^{\mathbb{C}}\right) .
$$

3.3. Uniform control on $\mathcal{R}_{\infty}$. As in Section 2.2, we make the change of coordinates $\alpha=\ln t$, for $\alpha \in(-\infty, \ln T]$. In these coordinates,

$$
\begin{aligned}
& \stackrel{\infty}{R_{i j k l}}=e^{-\alpha} R_{i j k l}, \\
& \stackrel{\infty}{R}_{i \alpha j \alpha}=e^{\alpha}\left(\Delta R_{i j}+2 R_{i k j l} R^{k l}-R_{i}^{k} R_{j k}+\frac{R_{i j}}{2 t}-\frac{1}{2} \nabla_{i} \nabla_{j} R\right), \\
& \stackrel{\infty}{R}_{i j \alpha k}=\nabla_{i} R_{j k}-\nabla_{j} R_{i k} .
\end{aligned}
$$

Define a metric $h$ on $\mathcal{M} \times(-\infty, \ln T]$ by

$$
h:=g\left(e^{\alpha}\right)+d \alpha^{2} .
$$

Following the perturbation of the Harnack quadratic performed by Hamilton (cf. [10] p. 239) and Lemma 7 in [2] by Brendle, we will modify the algebraic curvature tensor $\mathcal{R}_{\infty}$ to another algebraic curvature tensor, which lies in the interior of the relevant cone.

Proposition 3.5 (cf. 2, Lemma 7]). Let $V$ be a vector space of dimension $n+1$ and let $K \subset \operatorname{Sym}_{B}^{2}\left(\Lambda^{2} V^{*}\right)$ be a $G L(V)$-invariant convex cone containing all weakly positive definite curvature forms. Suppose $g(t)$ is a Ricci flow on a manifold $\mathcal{M}^{n}$ for $t \in[0, T]$ with derivatives up to order two of its curvature uniformly bounded. 
If $\mathcal{R}((\mathcal{M}, g(t)) \times \mathbb{R}) \in K$ for all $t \in[0, T]$, then there exists $C_{0}<\infty$ such that

$$
\mathcal{R}_{\infty}+\eta h \odot h \in \text { interior }(K)
$$

throughout $\check{\mathcal{M}}$ for $\eta \geq C_{0}$, where $\odot$ refers to the Kulkarni-Nomizu product.

Proof. By hypothesis, $\mathcal{R}((\mathcal{M}, g(t)) \times \mathbb{R}) \in K$ and therefore $\frac{1}{t} \mathcal{R}((\mathcal{M}, g(t)) \times \mathbb{R}) \in K$ for $t>0$. But

$$
\mathcal{R}_{\infty}-\frac{1}{t} \mathcal{R}((\mathcal{M}, g(t)) \times \mathbb{R})=\mathcal{R}_{\infty}-e^{-\alpha} \mathcal{R}((\mathcal{M}, g(t)) \times \mathbb{R})
$$

is bounded when measured with respect to $h$ (for $\alpha \leq \ln T$ ) by inspection of (3.8).

Therefore for large enough $\eta, \mathcal{R}_{\infty}-\frac{1}{t} \mathcal{R}((\mathcal{M}, g(t)) \times \mathbb{R})+\eta h \odot h$ is positive definite (viewed as a bilinear form as usual) and is thus in the interior of the cone $K$ by hypothesis. The result follows.

The above proposition could be considered a first step to obtaining the result with $\eta=0$ in particular cases - i.e. obtaining a Harnack inequality. Moreover, it allows us to compare the quadratic quantities $Q$ and $F$, given by (2.6) and (3.3) respectively, at $\mathcal{R}_{\infty}$ and at the perturbed $\mathcal{R}_{\infty}+\eta h \odot h$ whenever the latter hits the boundary of $K$ as follows:

Corollary 3.6 (cf. Brendle [2]). In the setting of Proposition 3.5, there exists $M_{2} \leq$ $\infty$ such that the following is true. If at some point in $\check{\mathcal{M}}$ we have $\mathcal{R}_{\infty}+\eta h \odot h \in \partial K$ for some $\eta>0$, then at that point we have

$$
Q\left(\mathcal{R}_{\infty}+\eta h \odot h, \check{g}_{\infty}\right)-Q\left(\mathcal{R}_{\infty}, \check{g}_{\infty}\right) \leq M_{2} \eta t h \odot h
$$

and

$$
F\left(\mathcal{R}_{\infty}+\eta h \odot h, \check{g}_{\infty}\right)-F\left(\mathcal{R}_{\infty}, \check{g}_{\infty}\right) \leq M_{2} \eta t h \odot h .
$$

The corollary follows from Proposition 3.5 because that proposition ensures that $\eta$ cannot be too large (or $\mathcal{R}_{\infty}+\eta h \odot h$ could not be on the boundary of $K$ ) and we may then use the fact that $Q$ and $F$ are both quadratic.

3.4. Perturbing $\mathcal{R}_{\infty}$. As is typical with a maximum principle proof, we must consider the evolution of a slightly perturbed version of $\mathcal{R}_{\infty}$ in order to proceed via a contradiction argument. (In this respect we also follow 10 and 2 .)

First we note that $h$ defined in (3.9) satisfies

$$
\mathcal{L}_{t \frac{\partial}{\partial t}} h=\mathcal{L}_{\frac{\partial}{\partial \alpha}} h=t \frac{\partial g}{\partial t}=-2 t \operatorname{Ric}(g(t)),
$$

and a short computation yields

$$
\stackrel{\infty}{\nabla}_{i} h=\left(t R_{i j}+\frac{1}{2} g_{i j}\right)\left(d \alpha \otimes d x^{j}+d x^{j} \otimes d \alpha\right),
$$

and using (3.1),

$$
\Delta_{t} h=\frac{t}{2}(d R \otimes d \alpha+d \alpha \otimes d R)+\frac{1}{2} \mid 2 t \text { Ric }+\left.g\right|^{2} d \alpha \otimes d \alpha .
$$

Consequently, if we have a uniform curvature bound, and a uniform bound on the first derivative of $R$ (e.g. in the setting of Proposition 3.5), then

$$
\left|\mathcal{L}_{t \frac{\partial}{\partial t}} h\right|_{h} \leq C t ; \quad\left|\Delta_{t} h\right|_{h} \leq C .
$$

We now require a 'defining function' $\varphi$ as in the work of Hamilton and Brendle. 
Lemma 3.7 (Hamilton [10] and Brendle [2]). Let $\left(\mathcal{M}^{n}, g(t)\right), t \in[0, T]$, with $T<\infty$, be a complete solution of the Ricci flow such that $|\mathcal{R}(g(t))|$ and $|\nabla \operatorname{Ric}(g(t))|$ are bounded on $\mathcal{M} \times[0, T]$ by $C_{0} \geq 0$. Then there exists a function $\varphi \in C^{\infty}(\mathcal{M})$ and $C=C\left(n, C_{0}, T\right)<\infty$ with the properties:

(1) $\varphi(x) \rightarrow \infty$ as $x \rightarrow \infty$,

(2) $\varphi(x) \geq 1$ for all $x \in \mathcal{M}$,

(3) $\sup _{\mathcal{M} \times[0, T]}|\nabla \varphi|_{g(t)} \leq C$,

(4) $\sup _{\mathcal{M} \times[0, T]}\left|\Delta_{g(t)} \varphi\right| \leq C$.

Using $\varphi$, we define

$$
\Upsilon:=\varphi h \odot h,
$$

and by (3.11) and (3.10),

$$
\left|\mathcal{L}_{t \frac{\partial}{\partial t}} \Upsilon\right|_{h} \leq C t ; \quad\left|\Delta_{t} \Upsilon\right|_{h} \leq C .
$$

Therefore there exists $M_{1}>0$ such that

$$
\mathcal{L}_{t \frac{\partial}{\partial t}} \Upsilon-t \Delta_{t} \Upsilon+t M_{1} \Upsilon \geq 0 .
$$

Lemma 3.8. In the setting of Proposition 3.5, there exists $\lambda \in(0, \infty)$ such that for all $\varepsilon>0$, if we define a tensor $\Theta$ on $\check{\mathcal{M}}$ by

$$
\Theta:=t \mathcal{R}_{\infty}+\varepsilon e^{\lambda t} \Upsilon
$$

then at any point $(x, t) \in \check{\mathcal{M}}$ where $\Theta$ is on the boundary of $K$, we have

$$
\mathcal{L}_{t \frac{\partial}{\partial t}} \Theta-t \Delta_{t} \Theta>\frac{1}{t} Q\left(\Theta, \check{g}_{\infty}\right)+\frac{1}{t} F\left(\Theta, \check{g}_{\infty}\right) .
$$

Moreover, there exist $\delta>0$ and $\Omega \Subset \mathcal{M}$ so that at any point $(x, t) \in \check{\mathcal{M}}$ with $t \leq \delta$ or $x \notin \Omega$, we have $\Theta(x, t) \in$ interior $(K)$.

Note that for a bilinear form $B$ on a vector space $V$, we write $B>0$ if $B(v, v)>0$ for all nonzero $v \in V$.

Proof. For initially arbitrary $\lambda>0$ and $\varepsilon>0$, take the corresponding $\Theta$ and suppose that $\Theta(x, t) \in \partial K$. By Corollary [3.6] we have

$$
\frac{1}{t} Q\left(\Theta, \check{g}_{\infty}\right)-t Q\left(\mathcal{R}_{\infty}, \check{g}_{\infty}\right) \leq M_{2} \varepsilon t e^{\lambda t} \Upsilon,
$$

and the same for $F$. By computing

$$
\mathcal{L}_{t \frac{\partial}{\partial t}} \Theta=t \mathcal{R}_{\infty}+t \mathcal{L}_{t \frac{\partial}{\partial t}} \mathcal{R}_{\infty}+\lambda t \varepsilon e^{\lambda t} \Upsilon+\varepsilon e^{\lambda t} \mathcal{L}_{t \frac{\partial}{\partial t}} \Upsilon
$$

and (using Lemma 3.2)

$$
\Delta_{t} \Theta=t \Delta_{t} \mathcal{R}_{\infty}+\varepsilon e^{\lambda t} \Delta_{t} \Upsilon
$$

we find, using (3.7), (3.13) and (3.15), that

$$
\begin{aligned}
\mathcal{L}_{t \frac{\partial}{\partial t}} \Theta-t \Delta_{t} \Theta & =\lambda t \varepsilon e^{\lambda t} \Upsilon+\varepsilon e^{\lambda t}\left(\mathcal{L}_{t \frac{\partial}{\partial t}} \Upsilon-t \Delta_{t} \Upsilon\right)+t F\left(\mathcal{R}_{\infty}, \check{g}_{\infty}\right)+t Q\left(\mathcal{R}_{\infty}, \check{g}_{\infty}\right) \\
& \geq \varepsilon t e^{\lambda t} \Upsilon\left(\lambda-M_{1}-2 M_{2}\right)+\frac{1}{t} Q\left(\Theta, \check{g}_{\infty}\right)+\frac{1}{t} F\left(\Theta, \check{g}_{\infty}\right) .
\end{aligned}
$$

Therefore, if $\lambda$ is chosen large enough, we have (3.14) as desired.

Finally, since

$$
\Theta=t\left(\mathcal{R}_{\infty}+\frac{\varepsilon e^{\lambda t} \varphi}{t} h \odot h\right),
$$


by Proposition 3.5 (taking $C_{0}$ from that proposition), if $t \leq \frac{\varepsilon}{C_{0}}=: \delta$ or $\varphi(x) \geq \frac{C_{0} T}{\varepsilon}$ (i.e. $\left.x \notin \varphi^{-1}\left(\left[1, \frac{C_{0} T}{\varepsilon}\right)\right)=: \Omega\right)$, then $\Theta(x, t) \in$ interior $(K)$, and we deduce the final part of the lemma.

Notice that the last claim of the previous lemma effectively gives us the initial condition for the maximum principle argument we will use to prove Theorem 2.7 in fact, it ensures that $\Theta$ (which should be regarded as a slight perturbation of $\mathcal{R}_{\infty}$ ) is in the cone for small enough $t$. Furthermore, it says nothing bad can happen at spatial infinity; i.e., if we were to fall out of the cone, it would happen within some compact region.

3.5. Proof of Theorem 2.7. The Ricci flow of the theorem has uniformly bounded curvature by Remark 2.8, and so by Shi's derivative estimates, for any $\delta>0$ we may assume that all derivatives of the curvature are bounded for $t \in[\delta, T]$ (with bounds depending on $\delta$ ). Therefore, it suffices to prove the result assuming that the Ricci flow exists on a closed time interval $[0, T]$ and that all derivatives of the curvature are bounded on $[0, T]$, since we could apply that result to $g(\delta+t)$ for $t \in[0, T-\delta]$ and then let $\delta \downarrow 0$.

We will apply the results we have established so far in Section 3 in the case that $K$ is the closed cone $\mathcal{C}\left(S_{k}\right)$ (as discussed in Appendix $\mathrm{B}$ ) on an $(n+1)$-dimensional vector space. We begin by choosing $\lambda$ as in Lemma 3.8. For each $\varepsilon>0$, we can then define the corresponding $\Theta$. Since our goal is to show that $\mathcal{R}_{\infty} \in \mathcal{C}\left(S_{k}\right)$, all we have to do is to show that $\Theta$ lies in the cone $\mathcal{C}\left(S_{k}\right)$ everywhere, for this arbitrarily small $\varepsilon$.

By the second part of Lemma 3.8, we know that $\Theta$ lies in the interior of the cone $\mathcal{C}\left(S_{k}\right)$ for small $t$ and also, outside $\Omega$ for all time.

Suppose, contrary to what we wish to establish, that, at some point, $\Theta$ fails to lie in the cone. Then we can pick a point $\left(x_{1}, t_{1}\right)$ with $t_{1}>0$ such that $\Theta\left(x_{1}, t_{1}\right)$ is on the boundary of the cone, and without loss of generality, we may assume that $t_{1}$ is the least possible time at which we could find such a point.

Since $\Theta\left(x_{1}, t_{1}\right)$ is on the boundary of the cone $\mathcal{C}\left(S_{k}\right)$, we can pick a nonzero element $\omega \in S_{k} \subset \Lambda^{2}\left(T_{\left(x_{1}, t_{1}\right)}^{\mathbb{C}} \check{\mathcal{M}}\right)$ such that $\Theta(\omega, \bar{\omega})=0$. By Corollary 3.4 since the cone is $G L(n+1, \mathbb{R})$-invariant, we have, at $\left(x_{1}, t_{1}\right)$,

$$
F\left(\Theta, \check{g}_{\infty}\right)(\omega, \bar{\omega})=0
$$

and by Theorem B.1 we have

$$
Q\left(\Theta, \check{g}_{\infty}\right)(\omega, \bar{\omega}) \geq 0
$$

so by Lemma 3.8 ,

$$
\left(\mathcal{L}_{t \frac{\partial}{\partial t}} \Theta-t \Delta_{t} \Theta\right)(\omega, \bar{\omega})>0 .
$$

We now extend $\omega$ to a neighbourhood of $x$ in $\mathcal{M}$ by parallel translation along radial geodesics using $\stackrel{\infty}{\nabla}$, and then extend $\omega$ in time to make it constant in the sense that

$$
\mathcal{L}_{t \frac{\partial}{\partial t}} \omega=0 \text {. }
$$

By construction,

$$
\Delta_{t} \omega=0
$$


at $\left(x_{1}, t_{1}\right)$. Since parallel translation of $\omega$ preserves its rank, we have $\operatorname{rank}(\omega) \leq k$ in a neighbourhood of $\left(x_{1}, t_{1}\right)$.

Next we define a function $f$ in that neighbourhood of $\left(x_{1}, t_{1}\right)$ by

$$
f:=\Theta(\omega, \bar{\omega}) .
$$

By definition of $f$, we have $f \geq 0$ near $x_{1}$ up to time $t_{1}$, and $f\left(x_{1}, t_{1}\right)=0$, and therefore at $\left(x_{1}, t_{1}\right)$ we have $\frac{\partial f}{\partial t} \leq 0$ and $\Delta_{g(t)} f \geq 0$, so that

$$
\frac{\partial f}{\partial t}-\Delta_{g(t)} f \leq 0 \text {. }
$$

On the other hand, we can compute at $\left(x_{1}, t_{1}\right)$,

$$
t \frac{\partial f}{\partial t}=\mathcal{L}_{t \frac{\partial}{\partial t}} \Theta(\omega, \bar{\omega})+\Theta\left(\mathcal{L}_{t \frac{\partial}{\partial t}} \omega, \bar{\omega}\right)+\Theta\left(\omega, \mathcal{L}_{t \frac{\partial}{\partial t}} \bar{\omega}\right)=\mathcal{L}_{t \frac{\partial}{\partial t}} \Theta(\omega, \bar{\omega})
$$

by (3.17), and

$$
\Delta_{t} f=\left(\Delta_{t} \Theta\right)(\omega, \bar{\omega})+\Theta\left(\Delta_{t} \omega, \bar{\omega}\right)+\Theta\left(\omega, \Delta_{t} \bar{\omega}\right)=\left(\Delta_{t} \Theta\right)(\omega, \bar{\omega})
$$

by (3.18). Therefore, by Lemma 3.2 and (3.16), we have at $\left(x_{1}, t_{1}\right)$,

$$
\frac{\partial f}{\partial t}-\Delta_{g(t)} f=\frac{1}{t}\left(\mathcal{L}_{t \frac{\partial}{\partial t}} \Theta-t \Delta_{t} \Theta\right)(\omega, \bar{\omega})>0,
$$

contradicting (3.19), and completing the argument.

\section{Appendix A. Computations}

In this appendix we give the formulae for the connection and curvature associated to the metric $\check{g}$ from Theorem 2.1 We also compute the Hessian of $-\frac{N}{2 t}$, thus justifying the assertions of Theorem 2.1

Proposition A.1. In the setting of Theorem 2.1, if $\Gamma_{j k}^{i}$ are the Christoffel symbols of $g(t)$ at some point $x \in \mathcal{M}$, then the Christoffel symbols of $\check{g}$ at $(x, t)$ are given by

$$
\begin{gathered}
\check{\Gamma}_{j k}^{i}=\Gamma_{j k}^{i} ; \quad \check{\Gamma}_{j 0}^{i}=-\left(R^{i}{ }_{j}+\frac{\delta^{i}{ }_{j}}{2 t}\right) ; \quad \check{\Gamma}_{00}^{i}=-\frac{1}{2} g^{i j} \frac{\partial R}{\partial x^{j}} ; \\
\check{\Gamma}_{j k}^{0}=\check{g}_{00}^{-1}\left(\frac{R_{j k}}{t}+\frac{g_{j k}}{2 t^{2}}\right) ; \quad \check{\Gamma}_{i 0}^{0}=\frac{1}{2 t} \check{g}_{00}^{-1} \frac{\partial R}{\partial x^{i}} ; \quad \check{\Gamma}_{00}^{0}=-\frac{3}{2 t}+\frac{\check{g}_{00}^{-1}}{2 t}\left[\frac{2 R}{t}+\mathbb{R}_{t}+\frac{n}{2 t^{2}}\right] .
\end{gathered}
$$

This proposition follows from the definition of the Christoffel symbols:

$$
\check{\Gamma}_{b c}^{a}:=\frac{1}{2} \check{g}^{a d}\left(\frac{\partial \check{g}_{c d}}{\partial x^{b}}+\frac{\partial \check{g}_{b d}}{\partial x^{c}}-\frac{\partial \check{g}_{b c}}{\partial x^{d}}\right),
$$

where $a, b, c, d$ are arbitrary indices, and the equation of Ricci flow. Using the standard formula for the coefficients of the curvature tensor,

$$
\check{R}_{a b c}{ }^{d}=\frac{\partial \check{\Gamma}_{a c}^{d}}{\partial x^{b}}-\frac{\partial \check{\Gamma}_{b c}^{d}}{\partial x^{a}}+\check{\Gamma}_{a c}^{e} \check{\Gamma}_{b e}^{d}-\check{\Gamma}_{b c}^{e} \check{\Gamma}_{a e}^{d}
$$

(where our sign convention is that $R_{1212} \geq 0$ on a positively curved manifold) and the equation for the evolution of Ric under Ricci flow,

$$
\frac{\partial R_{i}^{j}}{\partial t}=\Delta R_{i}^{j}+2 R^{j}{ }_{n i m} R^{m n}
$$

(see for example [18), we can then verify the formulae of the following proposition. 
Proposition A.2. In the setting of Theorem 2.1, the coefficients of the curvature tensor $\mathcal{R}$ of $\check{g}$ at $(x, t)$ are given by

$$
\begin{aligned}
\check{R}_{i j k l}= & \frac{1}{t} R_{i j k l}-\frac{\check{g}^{00}}{2 t^{2}}\left[\left(\operatorname{Ric}+\frac{g}{2 t}\right) \odot\left(\operatorname{Ric}+\frac{g}{2 t}\right)\right]_{i j k l} \\
= & \frac{1}{t} R_{i j k l}-\frac{\check{g}^{00}}{t^{2}}\left[R_{i k} R_{j l}-R_{i l} R_{j k}+\frac{1}{2 t}\left(R_{i k} g_{j l}+R_{j l} g_{i k}-R_{i l} g_{j k}-R_{j k} g_{i l}\right)\right. \\
& \left.\quad+\frac{1}{4 t^{2}}\left(g_{i k} g_{j l}-g_{i l} g_{j k}\right)\right], \\
\check{R}_{i 0 j 0}= & \frac{1}{t}\left(\Delta R_{i j}+2 R_{i k j l} R^{k l}-R_{i}^{k} R_{j k}+\frac{R_{i j}}{2 t}-\frac{1}{2} \nabla_{i} \nabla_{j} R\right) \\
& +\frac{\check{g}^{00}}{2 t^{2}}\left[\frac{1}{2} \nabla_{i} R \nabla_{j} R-\left(\frac{2 R}{t}+\partial_{t} R+\frac{n}{2 t^{2}}\right)\left(R_{i j}+\frac{g_{i j}}{2 t}\right)\right], \\
\check{R}_{i j 0 k}= & \frac{1}{t}\left(\nabla_{i} R_{j k}-\nabla_{j} R_{i k}\right)+\frac{\check{g}^{00}}{2 t^{2}}\left\{\left(R_{i k}+\frac{g_{i k}}{2 t}\right) \nabla_{j} R-\left(R_{j k}+\frac{g_{j k}}{2 t}\right) \nabla_{i} R\right\} .
\end{aligned}
$$

By taking the appropriate trace to give Ricci curvatures, and by using the formula for the coefficients of $\operatorname{Hess}_{\check{g}}(f)$,

$$
\check{\nabla}_{a b}^{2}(f)=\frac{\partial^{2} f}{\partial x^{a} \partial x^{b}}-\frac{\partial f}{\partial x^{c}} \check{\Gamma}_{a b}^{c},
$$

the equation for the evolution of $R$,

$$
R_{t}-\Delta R-2|\mathrm{Ric}|^{2}=0
$$

(see for example [18, Proposition 2.5.4]), and the contracted second Bianchi identity,

$$
\nabla_{i} R_{j}^{i}=\frac{1}{2} \nabla_{j} R
$$

we find:

Proposition A.3. In the setting of Theorem 2.1, the coefficients of the Ricci tensor Ric of $\check{g}$ at $(x, t)$ are given by

$$
\begin{aligned}
\check{R}_{i j}= & R_{i j}+\frac{\check{g}^{00}}{t}\left[M_{i j}-\left(R_{i j}+\frac{g_{i j}}{2 t}\right)\left(R+\frac{n}{2 t}\right)+\left(R_{i}^{k}+\frac{\delta_{i}^{k}}{2 t}\right)\left(R_{j k}+\frac{g_{j k}}{2 t}\right)\right] \\
& +\frac{\left(\check{g}^{00}\right)^{2}}{2 t^{2}}\left[\frac{1}{2} \nabla_{i} R \nabla_{j} R-\left(\frac{2 R}{t}+\partial_{t} R+\frac{n}{2 t^{2}}\right)\left(R_{i j}+\frac{g_{i j}}{2 t}\right)\right], \\
\check{R}_{i 0}= & \frac{\nabla_{i} R}{2}+\frac{\check{g}^{00}}{2 t}\left[\left(R_{i}^{j}+\frac{\delta_{i}^{j}}{2 t}\right) \nabla_{j} R-\left(R+\frac{n}{2 t}\right) \nabla_{i} R\right], \\
\check{R}_{00}= & \frac{\partial_{t} R}{2}+\frac{R}{2 t}+\frac{\check{g}^{00}}{2 t}\left[\frac{1}{2}|\nabla R|^{2}-\left(\frac{2 R}{t}+\partial_{t} R+\frac{n}{2 t^{2}}\right)\left(R+\frac{n}{2 t}\right)\right],
\end{aligned}
$$

where

$$
M_{i j}:=\Delta R_{i j}+2 R_{i k j l} R^{k l}-R_{i}^{k} R_{j k}+\frac{R_{i j}}{2 t}-\frac{1}{2} \nabla_{i} \nabla_{j} R .
$$


Moreover, we have

$$
\begin{aligned}
& \check{\nabla}_{i j}^{2}\left(-\frac{N}{2 t}\right)=-\left(R_{i j}+\frac{1}{2 t} g_{i j}\right)+\check{g}^{00}\left(\frac{R}{t}+\frac{n}{2 t^{2}}\right)\left(R_{i j}+\frac{1}{2 t} g_{i j}\right), \\
& \check{\nabla}_{i 0}^{2}\left(-\frac{N}{2 t}\right)=-\frac{\nabla_{i} R}{2}+\frac{\check{g}^{00}}{2} \nabla_{i} R\left(\frac{R}{t}+\frac{n}{2 t^{2}}\right), \\
& \check{\nabla}_{00}^{2}\left(-\frac{N}{2 t}\right)=-\frac{1}{2} \check{g}_{00}-\frac{\partial_{t} R}{2}-\frac{R}{2 t}+\frac{\check{g}^{00}}{2 t}\left(R+\frac{n}{2 t}\right)\left(\partial_{t} R+\frac{n}{2 t^{2}}+\frac{2 R}{t}\right) .
\end{aligned}
$$

By combining the formulae of Proposition A.3 and the definition of $\check{g}$, we deduce:

$$
\begin{aligned}
\check{R}_{i j}+\check{\nabla}_{i j}^{2}\left(-\frac{N}{2 t}\right)+\frac{1}{2} \check{g}_{i j} & =\frac{\check{g}^{00}}{t}\left(\Delta R_{i j}+2 R_{i k j l} R^{k l}+\frac{3 R_{i j}}{2 t}-\frac{1}{2} \nabla_{i} \nabla_{j} R+\frac{g_{i j}}{4 t^{2}}\right) \\
+ & \frac{\left(\check{g}^{00}\right)^{2}}{2 t^{2}}\left[\frac{1}{2} \nabla_{i} R \nabla_{j} R-\left(\frac{2 R}{t}+\partial_{t} R+\frac{n}{2 t^{2}}\right)\left(R_{i j}+\frac{g_{i j}}{2 t}\right)\right], \\
\check{R}_{i 0}+\check{\nabla}_{i 0}^{2}\left(-\frac{N}{2 t}\right)+\frac{1}{2} \check{g}_{i 0} & =\frac{\check{g}^{00}}{2 t}\left(R_{i}^{j}+\frac{\delta_{i}^{j}}{2 t}\right) \nabla_{j} R, \\
\check{R}_{00}+\check{\nabla}_{00}^{2}\left(-\frac{N}{2 t}\right)+\frac{1}{2} \check{g}_{00} & =\frac{\check{g}^{00}}{4 t}|\nabla R|^{2},
\end{aligned}
$$

and from these formulae it is easy to deduce Theorem 2.1.

\section{Appendix B. Wilking's COnes}

Let $\mathrm{V}$ be a vector space of dimension $m$. Given an algebraic curvature form $\mathcal{R} \in \operatorname{Sym}_{B}^{2}\left(\Lambda^{2} V^{*}\right)$, and an inner product $g$ on $V$, we wish to consider $Q(\mathcal{R}, g)$ defined as in (2.6), which is a vector in $S y m_{B}^{2}\left(\Lambda^{2} V^{*}\right)$.

In this section, the goal is to find closed convex cones within the space $\operatorname{Sym}_{B}^{2}\left(\Lambda^{2} V^{*}\right.$ ) (whose definition generally can use the inner product $g$ ) which are invariant under the ODE

$$
\frac{d \mathcal{R}}{d t}=Q(\mathcal{R}, g)
$$

(for fixed $g$ ) and invariant under the action of $O(V)$ (with respect to $g$ ). Thus we have a slightly more general setting than in Definition 2.12 and a combination of the so-called Uhlenbeck trick and Hamilton's ODE-PDE theorem (cf. 9]) says that these cones are invariant under Ricci flow on closed manifolds.

We now describe a special case of Wilking's unpublished method 21] for finding a large number of such cones 1

First, consider $V^{\mathbb{C}}$, the complexification of $V$, and extend each curvature operator/form complex linearly to act on $\Lambda^{2}\left(V^{\mathbb{C}}\right)$ as in 16 . (We will make such extensions implicitly in what follows.)

Using the inner product $g$, we can pick an orthonormal basis $\left\{e_{i}\right\}$ for $V$, and consider the corresponding action of $S O(m, \mathbb{C})$ on $V^{\mathbb{C}}$. Explicitly, an element $\sum v_{i} e_{i} \in V^{\mathbb{C}}$ (with $v_{i} \in \mathbb{C}$ ) would be mapped by $A \in S O(m, \mathbb{C}$ ) to the element $\sum_{i, j} A_{i j} v_{j} e_{i}$. This action then extends naturally to $\Lambda^{2}\left(V^{\mathbb{C}}\right)$.

\footnotetext{
${ }^{1}$ Added in proof: Since this work was completed, this method has been described in 22 .
} 
Theorem B.1 (Special case of Wilking [21]). Suppose $S \subset \Lambda^{2}\left(V^{\mathbb{C}}\right)$ is a subset (not necessarily a linear subspace) which is invariant under the action of $S O(m, \mathbb{C})$. Then the convex cone

$$
\mathcal{C}(S):=\left\{\mathcal{R} \in \operatorname{Sym}_{B}^{2}\left(\Lambda^{2} V^{*}\right) \mid \mathcal{R}(\omega, \bar{\omega}) \geq 0 \text { for all } \omega \in S\right\}
$$

is invariant under the $O D E$ (B.1). Equivalently, if $\mathcal{R} \in \partial \mathcal{C}(S)$ and we pick a nonzero $\omega \in S$ such that $\mathcal{R}(\omega, \bar{\omega})=0$, then $Q(\mathcal{R}, g)(\omega, \bar{\omega}) \geq 0$.

By taking different choices of $S$, Wilking recovered all the famous invariant curvature cones such as weakly positive isotropic curvature (WPIC) and its variants.

Note that the invariance of $S$ under the action of $S O(m, \mathbb{C})$ will depend on the inner product $g$, but not on the specific orthonormal basis we chose. For the purposes of this paper, we are interested in finding cones in $S y m_{B}^{2}\left(\Lambda^{2} V^{*}\right)$ which do not depend on $g$, and are therefore interested in finding sets $S$ which are invariant under the natural action of $G L(m, \mathbb{R})$ in addition to $S O(m, \mathbb{C})$.

Given an element $\omega \in \Lambda^{2}\left(V^{\mathbb{C}}\right)$, we define $\operatorname{rank}(\omega) \in\left\{0,1,2, \ldots,\left[\frac{m}{2}\right]\right\}$ to be the least number of simple elements $u \wedge v$ we need to sum to obtain $\omega$. We then define

$$
S_{k}=\left\{\omega \in \Lambda^{2}\left(V^{\mathbb{C}}\right) \mid \operatorname{rank}(\omega) \leq k\right\},
$$

and notice that it is both $G L(m, \mathbb{R})$ and $S O(m, \mathbb{C})$ invariant. By Theorem B.1. the curvature cones $\mathcal{C}\left(S_{k}\right)$, which are invariant under the action of $G L(m, \mathbb{R})$, are also invariant under the ODE (B.1) for one, and thus all $g$ (even degenerate weakly positive definite $g \in S^{2} m^{2} V$, by approximation).

As discussed in Section 2.4, one can check that $\mathcal{C}\left(S_{1}\right)$ is equal to a cone of curvature forms introduced by Brendle and Schoen [1. Meanwhile, since $\Lambda^{2} V \subset$ $S_{\left[\frac{m}{2}\right]}$, the cone $\mathcal{C}\left(S_{\left[\frac{m}{2}\right]}\right)$ consists of all weakly positive definite curvature forms, i.e. the cone of curvatures of manifolds of weakly positive curvature operator.

B.1. Proof of Wilking's result. For completeness, we give Wilking's proof of Theorem B.1. (Any inefficiencies or inaccuracies are due to us.) Using the basis $\left\{e_{i}\right\}$ for $V$, we have a (linear) isomorphism between $\Lambda^{2} V$ and $\mathfrak{s o}(m, \mathbb{R})$ - and hence between $\Lambda^{2}\left(V^{\mathbb{C}}\right)$ and $\mathfrak{s o}(m, \mathbb{C})$ - determined by

$$
e_{i} \wedge e_{j} \leftrightarrow\left[e_{i} \wedge e_{j}\right]_{k l}:=\delta_{i k} \delta_{j l}-\delta_{i l} \delta_{j k} .
$$

We will thus see curvature operators as bilinear forms, or operators, on $\mathfrak{s o}(m, \mathbb{R})$ or $\mathfrak{s o}(m, \mathbb{C})$ without change of notation. The action of $A \in S O(m, \mathbb{C})$ on $\Lambda^{2}\left(V^{\mathbb{C}}\right)$ then corresponds to the adjoint representation of $S O(m, \mathbb{C}), A d_{A}: \mathfrak{s o}(m, \mathbb{C}) \rightarrow \mathfrak{s o}(m, \mathbb{C})$, which can be written in terms of matrix multiplication as $A d_{A}(v)=A v A^{-1}$, so we may view $S$ as a subset of $\mathfrak{s o}(m, \mathbb{C})$ invariant under $A d$.

We will also need the adjoint representation $a d_{X}: \mathfrak{s o}(m, \mathbb{R}) \rightarrow \mathfrak{s o}(m, \mathbb{R})$ of $\mathfrak{s o}(m, \mathbb{R})$ (and the same for $\mathfrak{s o}(m, \mathbb{C}))$ :

$$
a d_{X} Y=\left.\frac{d}{d t} A d_{\exp (t X)} Y\right|_{t=0}=[X, Y]
$$

and the formula

$$
\left.\frac{d^{2}}{d t^{2}} A d_{\exp (t X)} Y\right|_{t=0}=a d_{X} a d_{X} Y .
$$

The natural inner product on $\mathfrak{s o}(m, \mathbb{R})$ is invariant under the adjoint representation of $S O(m, \mathbb{R})$ : for every $A \in S O(m, \mathbb{R})$,

$$
\langle X, Y\rangle=\left\langle A d_{A} X, A d_{A} Y\right\rangle
$$


for all $X, Y \in \mathfrak{s o}(m, \mathbb{R})$. By setting $A=\exp (t Z)$ in $(\underline{\mathrm{B} .4})$, for $Z \in \mathfrak{s o}(m, \mathbb{R})$, and differentiating with respect to $t$ at $t=0$ using (B.2), we know that

$$
0=\left\langle a d_{Z} X, Y\right\rangle+\left\langle X, a d_{Z} Y\right\rangle \text {. }
$$

The formula then extends to $X, Y, Z \in \mathfrak{s o}(m, \mathbb{C})$ with $a d$ the adjoint representation of $\mathfrak{s o}(n, \mathbb{C})$.

Recall (Hamilton 9, Böhm-Wilking 3]) that one can write

$$
Q(\mathcal{R}, g)=2\left(\mathcal{R}^{2}+\mathcal{R}^{\#}\right)
$$

where

$$
\mathcal{R}^{\#}(X, Y):=-\frac{1}{2} \operatorname{tr}\left(a d_{X} \circ \mathcal{R} \circ a d_{Y} \circ \mathcal{R}\right),
$$

the trace taking place over any orthonormal basis for $\mathfrak{s o}(m, \mathbb{R})$, or indeed over any unitary basis for $\mathfrak{s o}(m, \mathbb{C})$. Note that we are using the convention $-\operatorname{tr}(A B) / 2$ for the inner product on $\mathfrak{s o}(n, \mathbb{R})$ and the curvature operator which gives the identity for the unit round sphere. Therefore, since $\mathcal{R}^{2}$ is weakly positive definite, it suffices to prove that

$$
\mathcal{R}^{\#}(v, \bar{v}) \geq 0
$$

(extending bilinear forms complex linearly as usual) whenever $v \in S \subset \mathfrak{s o}(m, \mathbb{C})$ satisfies $\mathcal{R}(v, \bar{v})=0$, or equivalently

$$
-\operatorname{tr}\left(a d_{\bar{v}} \circ \mathcal{R} \circ a d_{v} \circ \mathcal{R}\right) \geq 0 .
$$

Because $\mathcal{R}(v, \bar{v})=0$ and $\mathcal{R}(w, \bar{w}) \geq 0$ for all $w \in S$ (and $S$ is invariant under $A d)$ we have

$$
0 \leq \mathcal{R}\left(A d_{\exp (t x)} v, A d_{\exp (t \bar{x}) \bar{v})}\right.
$$

for all $x \in \mathfrak{s o}(m, \mathbb{C})$, and differentiating twice with respect to $t$ using $(\underline{B .2})$ and (B.3) we find that

$$
0 \leq \mathcal{R}\left(a d_{x} a d_{x} v, \bar{v}\right)+\mathcal{R}\left(v, a d_{\bar{x}} a d_{\bar{x}} \bar{v}\right)+2 \mathcal{R}\left(a d_{x} v, a d_{\bar{x}} \bar{v}\right) .
$$

If we change $x$ to $i x$ to get a new inequality and then add the two inequalities, we get $0 \leq \mathcal{R}\left(a d_{x} v, a d_{\bar{x}} \bar{v}\right)$, and hence

$$
\mathcal{R}\left(a d_{v} x, a d_{\bar{v}} \bar{x}\right) \geq 0,
$$

for all $x \in \mathfrak{s o}(m, \mathbb{C})$. Note we have shown that by virtue of being on the boundary of $\mathcal{C}(S), \mathcal{R}$ is positive not only on $S$, but also on the entire image of $a d_{v}$.

Moreover, (B.6) and (B.5) imply

$$
0 \leq\left\langle\mathcal{R}\left(a d_{v} x\right), a d_{\bar{v}} \bar{x}\right\rangle=-\left\langle a d_{\bar{v}} \circ \mathcal{R} \circ a d_{v} x, \bar{x}\right\rangle,
$$

for all $x \in \mathfrak{s o}(m, \mathbb{C})$ and we find that $-a d_{\bar{v}} \circ \mathcal{R} \circ a d_{v} \geq 0$, or equivalently

$$
-a d_{v} \circ \mathcal{R} \circ a d_{\bar{v}} \geq 0,
$$

i.e. a positive definite Hermitian operator on $\mathfrak{s o}(m, \mathbb{C})$.

Thus we can diagonalise $-a d_{v} \circ \mathcal{R} \circ a d_{\bar{v}}$ on $\mathfrak{s o}(m, \mathbb{C})$, finding a unitary basis $\left\{\omega_{i}\right\}$ (i.e. $\left.\left\langle\omega_{i}, \bar{\omega}_{i}\right\rangle=\delta_{i j}\right)$ such that $-a d_{v} \circ \mathcal{R} \circ a d_{\bar{v}}\left(\omega_{i}\right)=\lambda_{i} \omega_{i}$, with $\lambda_{i} \geq 0$. For any $i$ such 
that $\lambda_{i}>0$, we therefore find that $\omega_{i}$ is in the image of $a d_{v}$, and thus $\mathcal{R}\left(\omega_{i}, \bar{\omega}_{i}\right) \geq 0$ by (B.6). Consequently,

$$
\begin{aligned}
-\operatorname{tr}\left(a d_{\bar{v}} \circ \mathcal{R} \circ a d_{v} \circ \mathcal{R}\right) & =-\operatorname{tr}\left(\mathcal{R} \circ a d_{v} \circ \mathcal{R} \circ a d_{\bar{v}}\right) \\
& =\sum_{i}\left\langle\mathcal{R} \circ a d_{v} \circ \mathcal{R} \circ a d_{\bar{v}}\left(\omega_{i}\right), \bar{\omega}_{i}\right\rangle \\
& =\sum_{i} \lambda_{i}\left\langle\mathcal{R}\left(\omega_{i}\right), \bar{\omega}_{i}\right\rangle \\
& =\sum_{i} \lambda_{i} \mathcal{R}\left(\omega_{i}, \bar{\omega}_{i}\right) \\
& \geq 0 .
\end{aligned}
$$

Remark B.2. Viewing $S$ as a subset of $\mathfrak{s o}(m, \mathbb{C})$ makes it easier to describe some further interesting examples (as observed by Wilking). The cone $\mathcal{C}(S)$ corresponding to $S=\left\{X \mid \operatorname{rank}(X)=2 ; X^{2}=0\right\}$, for example, is the cone of weakly positive isotropic curvature (WPIC) operators.

\section{ACKNOWLEDGEMENTS}

We thank Burkhard Wilking, Simon Brendle and Mario Micallef for useful conversations. Both authors were supported by The Leverhulme Trust. The first author was also partially supported by the DGI (Spain) and FEDER Project MTM2007-65852, and by the net REAG MTM2008-01013-E.

\section{REFERENCES}

[1] S. Brendle and R. Schoen, Manifolds with 1/4-pinched curvature are space forms. J. Amer. Math. Soc. 22 (2009) 287-307. MR2449060 (2010a:53045)

[2] S. Brendle, A generalization of Hamilton's differential Harnack inequality for the Ricci flow. J. Differential Geom. 82 (2009), 207-227. MR2504774 (2010d:53070)

[3] C. Böhm and B. Wilking, Manifolds with positive curvature operators are space forms. Ann. of Math. (2) 167 (2008), no. 3, 1079-1097. MR2415394 (2009h:53146)

[4] E. Cabezas-Rivas and P.M. Topping, The Canonical Shrinking Soliton associated to a Ricci flow. Calc. Var. and PDE 43 (2012), 173-184.

[5] B. Chow and S.-C. Chu, A geometric interpretation of Hamilton's Harnack inequality for the Ricci flow. Math. Res. Lett. 2 (1995) 701-718. MR1362964 (97f:53063)

[6] B. Chow and D. Knopf, New Li-Yau-Hamilton inequalities for the Ricci Flow via the spacetime approach. J. Differential Geom. 60 (2002), 1-54. MR.1924591|(2003g:53116)

[7] B. Chow, P. Lu and L. Ni, Hamilton's Ricci flow. Graduate Studies in Math. 77, Amer. Math. Soc., Providence, RI (2006). MR2274812 (2008a:53068)

[8] R. S. Hamilton, Three-manifolds with positive Ricci curvature. J. Differential Geom. 17 (1982) 255-306. MR664497 (84a:53050)

[9] R. S. Hamilton, Four-manifolds with positive curvature operator. J. Differential Geom. 24 (1986), no. 2, 153-179. MR862046 (87m:53055)

[10] R.S. Hamilton, The Harnack estimate for the Ricci flow. J. Differential Geom. 37 (1993) 225-243. MR 1198607 (93k:58052)

[11] R. S. Hamilton, The formation of singularities in the Ricci flow. Surveys in Differential Geometry, Vol. II (Cambridge, MA, 1993) 7-136, Internat. Press, Cambridge, MA, 1995. MR1375255(97e:53075)

[12] D. Knopf, Positivity of Ricci curvature under the Kähler-Ricci flow. Commun. Contemp. Math. 8 (2006), no. 1, 123-133. MR2208813(2006k:53114)

[13] S. Kobayashi and K. Nomizu, Foundations of differential geometry. Vol I. Interscience Publishers, New York-London (1963) xi+329 pp. MR0152974(27:2945)

[14] D. Máximo, Non-negative Ricci curvature on closed manifolds under Ricci flow. Proc. Amer. Math. Soc. 139 (2011), 675-685. MR 2736347 
[15] R.J. McCann and P.M. Topping, Ricci flow, entropy and optimal transportation. Amer. J. Math. 132 (2010), 711-730. MR2666905

[16] M.J. Micallef and J.D. Moore, Minimal two-spheres and the topology of manifolds with positive curvature on totally isotropic two-planes. Ann. of Math. (2) 127 (1988) 199-227. MR924677|(89e:53088)

[17] G. Perelman, The entropy formula for the Ricci flow and its geometric applications. http:// arXiv.org/abs/math/0211159v1 (2002).

[18] P.M. Topping, Lectures on the Ricci flow. London Math. Soc. Lecture Notes Series 325, Cambridge University Press, Cambridge, 2006. MR2265040 (2007h:53105)

[19] P.M. Topping, $\mathcal{L}$-optimal transportation for Ricci flow. J. Reine Angew. Math. 636 (2009) 93-122. MR 2572247

[20] P.M. Topping, Ricci Flow: The Foundations via Optimal Transportation. To appear in 'Séminaires et Congrès.' S.M.F. (2009) http://www.warwick.ac.uk/ maseq

[21] B. Wilking, private communication (2009).

[22] B. Wilking, A Lie algebraic approach to Ricci Flow invariant curvature conditions and Harnack inequalities. To appear in J. Reine Angew. Math. arXiv:1011.3561

Mathematisches Institut, Fachbereich Mathematik und Informatik der Universität Münster, Einsteinstrasse 62, 48149 Münster, Germany

Mathematics Institute, University of Warwick, Coventry, CV4 7AL, United Kingdom 